\title{
What is new in stroke imaging and intervention?
}

\author{
Authors: Philip White ${ }^{\mathrm{A}, \mathrm{B}}$ and Andrew Nanapragasam ${ }^{\mathrm{C}}$
}

Recent updates to guidelines around brain imaging in stroke and transient ischaemic attack are reviewed. A more detailed examination of advanced brain imaging in acute stroke is presented. The recent evidence for endovascular mechanical thrombectomy in acute stroke is reviewed. The implications of delivering thrombectomy are discussed.

KEYWORDS: Advanced imaging, angiography, CT brain, ischemic stroke, thrombectomy

\section{Introduction}

Recent years have seen significant changes in the imaging and treatment of stroke. As thrombolytic therapy revolutionised stroke services over the last 15 years, endovascular therapy promises to be just as transformative over the next 5-10 years.

\section{The use of brain imaging in transient ischaemic attack assessment}

The January 2017 National Institute for Health and Care Excellence (NICE) guidelines update to transient ischaemic attack (TIA) assessment reports that patients first require assessment by a specialist, with brain imaging only being requested if the specialist deems it may alter management. ${ }^{1}$ This is in accordance with the updated National clinical guideline for stroke (NCGS, 5th edn). ${ }^{2}$ If acute haemorrhage is the clinical concern then computerised tomography (CT) brain suffices.

\section{The use of brain imaging in acute stroke assessment}

For cases of suspected acute stroke, non-contrast CT (NCCT) brain examination is the mainstay of imaging as it is a reliable, universally available and cost-effective means of assessment. NCCT brain imaging can exclude stroke mimics and differentiate between an ischaemic and haemorrhagic stroke. For patients eligible for early treatment or those with signs of a moderate/ severe stroke, NICE and the NCGS have recommended that an

Authors: A professor of neuroradiology, Newcastle University, Newcastle upon Tyne, UK; ${ }^{B}$ honorary consultant neuroradiologist, Newcastle upon Tyne Hospitals NHS Trust, Newcastle upon Tyne, UK; C radiology ST2, Newcastle upon Tyne Hospitals NHS Trust, Newcastle upon Tyne, UK
NCCT brain exam is performed 'immediately' or at least within an hour of presentation. ${ }^{3}$

The diagnostic utility of NCCT brain imaging is well established, but it can also provide an indication of prognosis. The Alberta Stroke Programme Early CT Score (ASPECTS) is a means of deriving prognostic information for patients with an anterior circulation stroke. ${ }^{4}$ The cerebrum is divided into 10 topographic regions. For each region affected by an anterior circulation infarction, a point is deducted. The stroke-affected regions are best assessed with a narrow window width and window level, eg between 35 and 45 Hounsfield units. Good interobserver reproducibility is seen with this scoring method. ${ }^{5}$ A score of fewer than 8 is an indicator of an increased risk of symptomatic intracranial haemorrhage and of a worse outcome following intravenous thrombolysis.

\section{Advances in the imaging of acute ischaemic stroke}

Following the diagnosis of an acute ischaemic stroke, it is established practice to administer intravenous thrombolysis (IVT) within 4.5 hours of the onset of symptoms. ${ }^{6}$ However, IVT is not without its risks. In fact, a $6 \%$ increase in symptomatic intracerebral haemorrhage was noted by a Cochrane systematic review. $^{6}$

Is advanced brain imaging able to better select patients for treatment by identifying those who are at an increased risk of complications, such as symptomatic intracerebral haemorrhage? Could imaging also identify patients who may benefit from treatment despite not presenting within 4.5 hours of symptom onset?

Advanced brain imaging in stroke consists of all magnetic resonance imaging (MRI) and any form of CT imaging other than an NCCT brain scan.

\section{CT angiography}

CT angiography (CTA), from aortic arch to skull vertex, has a variety of clinical benefits as well as being quick and cheap to perform. Firstly, it adds diagnostic certainty to the NCCT brain scan. CTA can identify the location of the arterial occlusion, which is essential if proceeding on to endovascular therapy. ${ }^{7}$ Furthermore, the degree of any vessel stenosis can be identified, which is relevant for both the acute and long-term management of patients. ${ }^{8}$ A recent study based on multiple randomised controlled trials of individual patient record data found that use of IVT was associated with an improvement in functional outcome if CTA demonstrated arterial obstruction (Fig 1), but not if CTA was normal. ${ }^{9}$ If this result is corroborated by future research, ASPECTS or CTA could be used to spare some patients exposure to the risks 


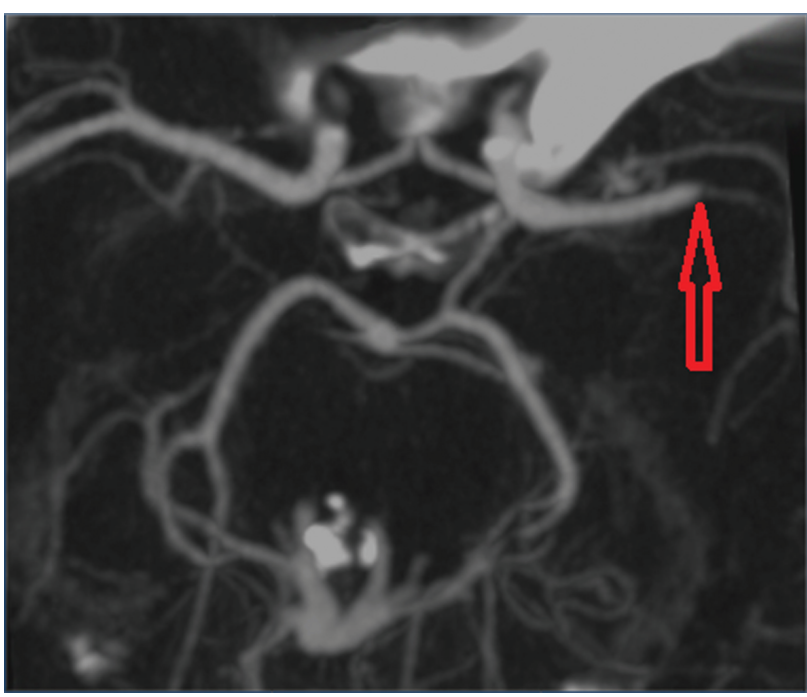

Fig 1. A maximum intensity projection in the axial plane from a computerised tomography angiogram demonstrating left middle cerebral artery occlusion (arrow)

of thrombolytics, if they are unlikely to benefit from their use. Despite symptoms and NCCT brain imaging consistent with an ischaemic stroke, many patients will have patent intracerebral vasculature on CTA, probably around $60 \%$. $^{10,11}$

The volume of ischaemic tissue can be estimated from CTA. Those patients with a large 'ischaemic core' receive less benefit from thrombolysis. Indeed, the presence of a large ischaemic core is considered to be a relative contraindication to thrombolysis. ${ }^{12}$

CTA is also able to detect the presence and extent of collateral blood supply. A multiphase CT angiogram allows even better delineation of collateral supply as it provides progressive, temporal information on the blood flow. ${ }^{13}$ A greater collateral blood supply may justify the extension of the reperfusion time window. Conversely, those patients with a lack of collateral blood vessels are unlikely to see benefit from endovascular therapy, and could be deemed ineligible for this treatment on these grounds. ${ }^{14}$

\section{CT perfusion}

CT perfusion imaging uses sequential brain imaging to evaluate the flow of contrast through cerebral vessels (mean transit time), the total cerebral blood volume and the cerebral blood flow to a region. With this information, the extent of reversible and irreversible ischaemic tissue can be estimated. Reversibly injured tissue can be estimated using a time to peak of greater than 6 seconds. ${ }^{15}$ The extent of irreversible damage can be assessed by identifying tissue with a $30 \%$ lower cerebral blood flow than the normal brain parenchyma. ${ }^{16}$ If the ischaemic core volume is greater than $70-100 \mathrm{~mL}$, then there is a low chance of functional outcome regardless of the treatment delivered. Care should be taken when interpreting CT perfusion scans in patients with confounding pathology, such as arrhythmias, low cardiac output or aortic valvular disease. The interpretation of CT perfusion scans can also be impaired by technical issues, such as patient movement, timing of the bolus and the spatial separation of slices.
MRI

Patients with signs of a stroke without evidence of ischaemia on $\mathrm{CT}$ can be imaged with diffusion-weighted MRI sequences as signs of ischaemia appear on MRI earlier than on CT imaging. Despite MRI being more sensitive to the early changes in ischaemic stroke, systematic review found that approximately one-third of patients with a clinical diagnosis of a 'minor stroke' were found to have no corroborative signs on MRI. ${ }^{17}$ For those patients who are not able to report the time of symptom onset, diffusionweighted MRI coupled with fluid-attenuated inversion recovery sequences may be able to time strokes accurately. ${ }^{18}$ The lack of CT evidence for ischaemia does not negate the requirement for treatment. Therefore, although an MRI to confirm ischaemia may be satisfying for clinicians, it is not a requirement for acute management. Furthermore, as MRI takes longer than a CT scan, it is less safe in the acutely unwell patient and more susceptible to motion artefacts.

As immediate access to MRI is limited in the UK, it is not a practical alternative to $\mathrm{CT}$ imaging in most stroke patients, but is required in some circumstances. ${ }^{2,7}$

\section{Positron emission tomography techniques}

The role of positron emission tomography (PET) techniques in the acute management of human stroke has been little studied. The severe time constraints in clinical practice make it extremely challenging to access PET imaging and these are therefore unlikely to enter routine clinical practice in the immediate future.

\section{The role of endovascular techniques in the treatment of acute ischaemic stroke}

IVT is least effective in large artery occlusive stroke where recanalisation rates are low. ${ }^{8,10,11}$ As a result, functional clinical outcomes in large artery occlusive stroke with IVT are disappointing - with an approximate $25 \%$ good functional outcome. ${ }^{19}$ Recent randomised trials have shown that there is no benefit with the use of intra-arterial thrombolysis compared with IVT. ${ }^{20,21}$

An individual patient record meta-analysis of five very recent trials (HERMES = Highly Effective Reperfusion evaluated in Multiple Endovascular Stroke trials), all of which used CTA for patient selection and employed modern devices for clot extraction, constitutes the best evidence so far for the use of endovascular clot extraction in acute ischaemic stroke (Fig 2). ${ }^{22}$ The key finding is that mechanical thrombectomy (MT) of proximal anterior circulation large artery occlusive strokes is associated with a significantly reduced disability after 90 days compared with standard medical treatment. In order to reduce disability by at least one level on the modified Rankin scale, the number needed to treat with endovascular therapy is only 2.6. Patients with an ASPECTS of $\geq 6$ were identified as a subgroup likely to experience a statistical benefit from endovascular treatment.

The significance of this meta-analysis should not be underestimated. Mechanical thrombectomy (using either stent retriever and/or aspiration techniques) combined with IVT can now be considered best practice for those previously functionally independent patients presenting within 6 hours of a large vessel anterior circulation occlusion, provided ASPECTS is $\geq 6$ and NIHSS 


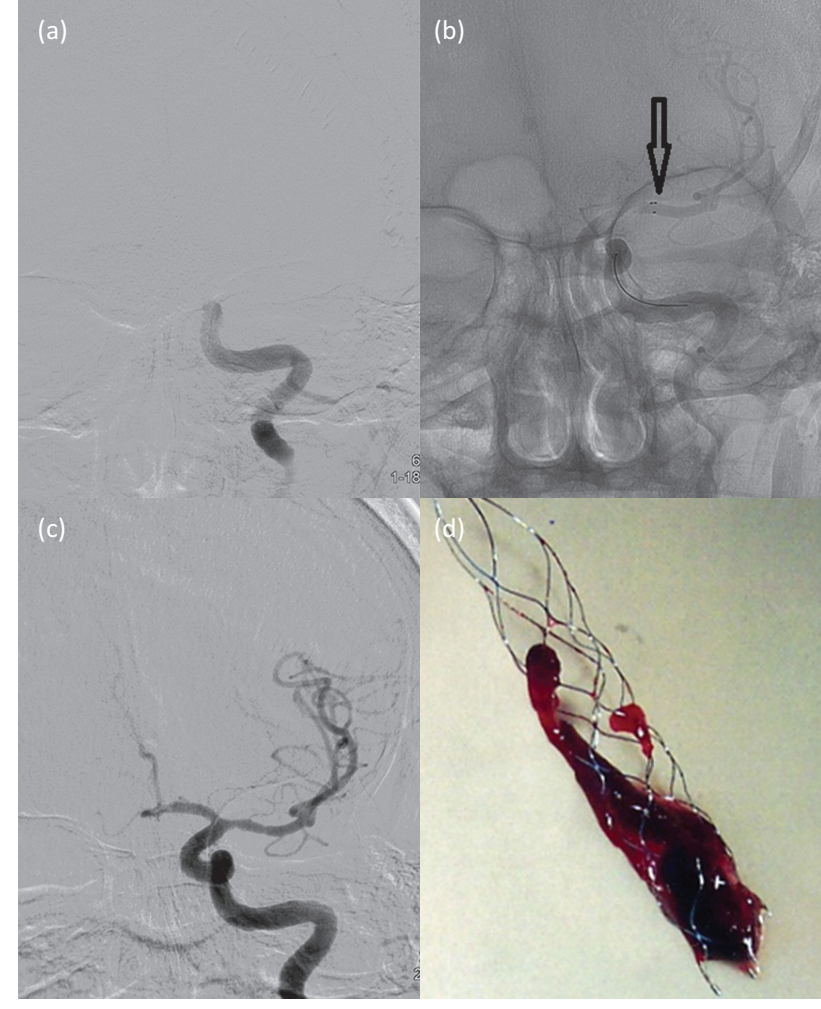

Fig 2. Digital subtraction cerebral angiogram before (a) and during (b) mechanical thrombectomy for combined left ICA/MCA occlusion With stent retriever deployed (arrow) the MCA is recanalised (b) and flow restored. Once stent retriever is removed along with the clot, normal reperfusion is restored (c) - complete recanalisation. The patient has woken up on the table and is now moving, hence blurring of image. Image $d$ demonstrates the extracted clot in the stent retriever. ICA = internal carotid artery; $\mathrm{MCA}=$ middle cerebral artery

(National Institutes of Health Stroke Scale) score is $\geq 6$. $^{2}$ Those patients with a contraindication to thrombolysis, but not to thrombectomy, will still benefit from endovascular treatment. ${ }^{22}$ Although MT has been shown to significantly improve outcomes up to 7.3 hours from the onset of symptoms, the relative benefit from intervention decreases progressively with time. ${ }^{2}$ The applicability of the technique in the NHS has been confirmed in a small UK trial (PISTE - Pragmatic Ischaemic Stroke Thrombectomy Evaluation trial), with the clinical/technical outcomes and process times for MT all in line with those seen in the HERMES metaanalysis. $^{22-24}$

Patients presenting beyond the 6-hour time window for MT may still benefit from endovascular treatment. These patients can be identified with the use of advanced brain imaging. Based on recent trials, the MT window for patients with proven advanced brain imaging features of good prognosis may be extended as far as $8-12$ hours. ${ }^{25,26}$

The use of MT for posterior circulation large artery occlusive strokes is under investigation. Its efficacy and potential role in treatment are promising, but yet to be confirmed. Questions pertaining to the optimum techniques for endovascular therapy also remain unanswered. The benefit of a balloon guide, the difference (if any) between primary aspiration and retrievable stents as a means of thrombectomy, and the relative advantages/disadvantages of general anaesthesia compared with conscious sedation are all topics of debate. Other areas where the MT data is equivocal and requires further evaluation include the treatment of patients with a large artery occlusive stroke accompanied only by minimal symptoms (ie NIHSS score <6), and the treatment of patients with small vessel occlusions. The time window for endovascular and intravenous treatment is also being examined by, among others, the DAWN and ECASS- 4 trials. $^{27}$

\section{Delivery of mechanical thrombectomy}

The imaging and intervention requirements to deliver MT will require substantial reconfiguration of stroke services. Streamlined pathways allowing patients in smaller hospitals to promptly access interventional services at larger centres are a likely solution. ${ }^{28}$ Up to $10 \%$ of stroke patients are eligible for MT under current evidence and that may be extended with additional trial data in the coming years. ${ }^{29}$ Investment is needed not only for the implementation of the established best practice, but also for the further investigation of uncertain aspects of endovascular therapy, particularly the precise roles and optimum techniques for advanced imaging. ${ }^{30}$

\section{Conclusions}

The evidence base regarding the imaging and endovascular treatment of acute ischaemic stroke has undergone a considerable recent revision. Trials have defined CTA as a key tool in the selection of patients for both IVT and MT. MT has unequivocal and significant benefits for an important subgroup of stroke patients. Further research in this rapidly evolving field will reveal refinements to the advances presented in this article. However, improvements in service delivery are first needed to fully realise the benefits of the work done thus far.

\section{Conflicts of interest}

PW is co-principal investigator for two randomised trials (PISTE and STABILISE) investigating different aspects of thrombectomy in acute stroke. Start-up phase of PISTE was mainly funded by the Stroke Association, but was also part-funded by unrestricted institutional educational grants from Covidien and Codman who both manufacture devices used for stroke thrombectomy. STABILISE is part-funded by Microvention. PW has also undertaken educational consultancy work within the last 3 years for Codman and Microvention, who both manufacture devices used for stroke thrombectomy. He is a member of the Intercollegiate Stroke Working party and NHS England Clinical Neurosciences Clinical Reference Group.

AN has no conflicts of interest to declare.

\section{References}

1 National Institute for Health and Care Excellence. Surveillance report 2017 - Stroke and transient ischaemic attach in over 16s: diagnosis and management (2008) NICE guideline CG68. London: NICE, 2017.

2 Intercollegiate Stroke Working Party. National clinical guideline for stroke, 5th edn. London: Royal College of Physicians, 2016.

3 National Institute for Health and Care Excellence. Acute stroke. London: NICE, 2017. https://pathways.nice.org.uk/pathways/ stroke/acute-stroke [Accessed 10 August 2017].

4 Barber PA, Demchuk AM, Zhang J, Buchan AM. Validity and reliability of a quantitative computed tomography score in predicting 
outcome of hyperacute stroke before thrombolytic therapy. ASPECTS Study Group. Alberta Stroke Programme Early CT Score. Lancet 2000;355:1670-4.

5 Finlayson O, John V, Yeung R et al. Interobserver agreement of ASPECT score distribution for noncontrast CT, CT angiography, and CT perfusion in acute stroke. Stroke 2013;44:234-6.

6 Wardlaw JM, Murray V, Berge E, del Zoppo GJ. Thrombolysis for acute ischaemic stroke. The Cochrane Database Syst Rev 2014; (7):CD000213.

7 White PM, Bhalla A, Dinsmore ] et al. Standards for providing safe acute ischaemic stroke thrombectomy services (September 2015). Clin Radiol 2017;72:175.e1-175.e9.

8 Menon BK, Qazi E, Nambiar V et al. Differential effect of baseline CTA Collaterals on Clinical Outcome in patients enrolled in the IMS-III trial. Stroke 2015;46:1239-44.

9 Mair G, von Kummer R, Adami A et al. Arterial obstruction on computed tomographic or magnetic resonance angiography and response to intravenous thrombolytics in ischemic stroke. Stroke 2017:48:353-60.

10 El Tawil S, Cheripelli B, Huang X et al. How many stroke patients might be eligible for mechanical thrombectomy? Eur Stroke ] 2016;1:264-71.

11 Smith WS, Lev MH, English JD et al. Significance of large vessel intracranial occlusion causing acute ischemic stroke and TIA. Stroke 2009;40:3834-40.

12 Wardlaw JM, Mielke O. Early signs of brain infarction at CT: observer reliability and outcome after thrombolytic treatmentsystematic review. Radiology 2005;235:444-53.

13 Menon BK, d'Esterre CD, Qazi EM et al. Multiphase CT angiography: a new tool for the imaging triage of patients with acute ischemic stroke. Radiology 2015;275:510-20.

14 Elijovich L, Goyal N, Mainali S et al. CTA collateral score predicts infarct volume and clinical outcome after endovascular therapy for acute ischemic stroke: a retrospective chart review. J Neurointerv Surg 2016:8:559-62.

15 Bivard A, Levi C, Spratt N, Parsons M. Perfusion CT in acute stroke: a comprehensive analysis of infarct and penumbra. Radiology 2013;267:543-50.

16 Campbell BC, Christensen S, Levi CR et al. Cerebral blood flow is the optimal CT perfusion parameter for assessing infarct core. Stroke 2011:42:3435-40.

17 Wardlaw J, Brazzelli M, Miranda $\mathrm{H}$ et al. An assessment of the cost-effectiveness of magnetic resonance, including diffusionweighted imaging, in patients with transient ischaemic attack and minor stroke: a systematic review, meta-analysis and economic evaluation. Health Technol Assess 2014;18:1-368.
18 Aoki J, Kimura K, Iguchi $Y$ et al. FLAIR can estimate the onset time in acute ischemic stroke patients. J Neurol Sci 2010:293:39-44

19 Campbell BV, Hill MD, Rubiera M et al. Safety and efficacy of Solitaire stent thrombectomy Individual patient data meta - analysis of randomized trials. Stroke 2016;14:798-806.

20 Ciccone A, Valvassori L, Ponzio M et al. Intra-arterial or intravenous thrombolysis for acute ischemic stroke? The SYNTHESIS pilot trial. J Neurointerv Surg 2010;2:74-9.

21 Nam J, Jing H, O'Reilly D. Intra-arterial thrombolysis vs. standard treatment or intravenous thrombolysis in adults with acute ischemic stroke: a systematic review and meta-analysis. Int I Stroke 2015;10:13-22.

22 Goyal M, Menon BK, van Zwam WH et al. Endovascular thrombectomy after large-vessel ischaemic stroke: a meta-analysis of individual patient data from five randomised trials. Lancet 2016;387:1723-31.

23 Saver JL, Goyal M, van der Lugt A et al. Time to treatment with endovascular thrombectomy and outcomes from ischemic stroke: a meta-analysis. JAMA 2016;316:1279-88.

24 Muir KW, Ford GA, Messow CM et al. Endovascular therapy for acute ischaemic stroke: the Pragmatic Ischaemic Stroke Thrombectomy Evaluation (PISTE) randomised, controlled trial. J Neurol 2017:88:38-44.

25 Jovin TG, Chamorro A, Cobo E et al. Thrombectomy within 8 hours after symptom onset in ischemic stroke. N Engl J Med 2015;372:2296-306.

26 Goyal M, Demchuk AM, Menon BK et al. Randomized assessment of rapid endovascular treatment of ischemic stroke. N Engl J Med 2015:372:1019-30.

27 Amiri $\mathrm{H}$, Bluhmki E, Bendszus $\mathrm{M}$ et al. European cooperative acute stroke study-4: extending the time for thrombolysis in emergency neurological deficits ECASS-4: ExTEND. Int ] Stroke 2016;11:260-7.

28 Milne MS, Holodinsky JK, Hill MD et al. Drip 'n ship versus mothership for endovascular treatment: modeling the best transportation options for optimal outcomes. Stroke 2017:48:791-4.

29 Flynn D, Ford G, McMeekin P et al. Future delivery of thrombectomy services in England: a Delphi study with stroke physicians [abstract]. Liverpool: UK Stroke Forum Conference, 28-30 November 2016.

30 Muir KW, White P. HERMES: messenger for stroke interventional treatment. Lancet 2016;387:1695-7.

Address for correspondence: Professor Phil White, Institute of Neuroscience (Stroke Research Group), Newcastle University, Newcastle Upon Tyne NE2 4AE, UK.

Email: phil.white@ncl.ac.uk 